
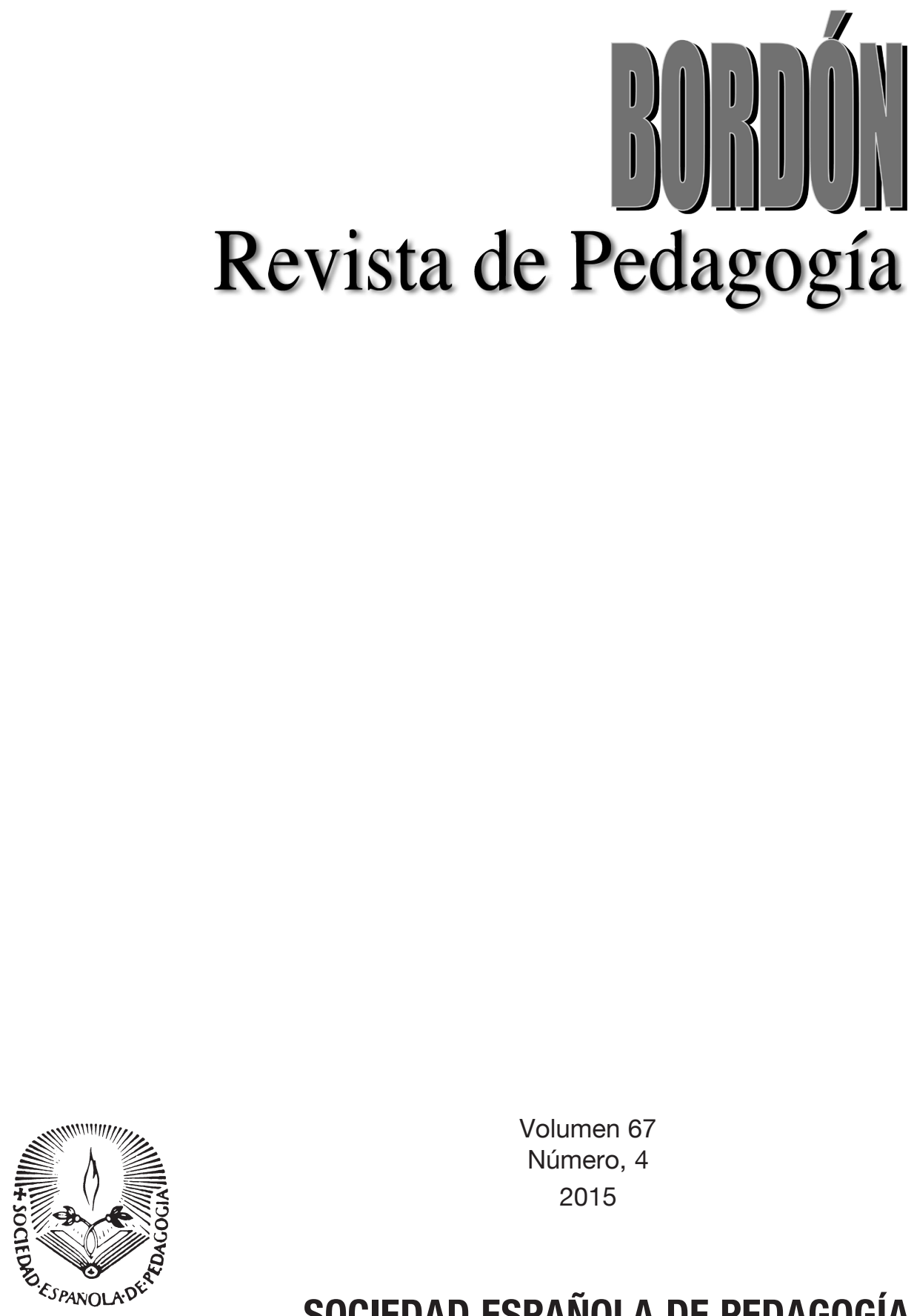

Volumen 67

Número, 4

2015

SOCIEDAD ESPAÑOLA DE PEDAGOGÍA 


\title{
MUJER Y CAPITAL SOCIAL EN LAS COMUNIDADES PESQUERAS DE CABO UERDE
}

\section{Women associal capital in the Cape Uerde fishing communities}

\author{
SIMAO PAULO RODRIGUES VARELA \\ Universidade Jean Piaget-Cabo Verde
}

MIGUEL A. SANTOS REGOY MAR LORENZO MOLEDO

Universidad de Santiago de Compostela

D01: 10.13042/Bordon.2015.67408

Fecha de recepción: 01/09/2014 - Fecha de aceptación: 31/03/2015

Autor de contacto / Corresponding Author: Miguel A. Santos Rego. E-mail: miguelangel.santos@usc.es

INTRODUCCIÓN. Lo que se pretende en este trabajo es contribuir a reducir el desequilibrio en la atención prestada por la investigación sobre el capital social (CS) a las redes asociativas en detrimento de las femeninas. Para ello nos valemos de un estudio sobre el terreno en Cabo Verde, centrado en las comunidades pesqueras de la isla de Santiago. MÉTODO. Hicimos uso de una metodología cuantitativa de corte descriptivo de encuesta empleando un cuestionario. La muestra se compuso de 340 personas, seleccionadas al azar, entre los miembros de las comunidades cuyas edades iban de los 15 a los 64 años. Para medir el Índice de Capital Social (ICS) se tuvieron en cuenta variables relacionadas con percepciones de confianza, redes, solidaridad, inclusión social y acción voluntaria. RESULTADOS. No se dan diferencias estadísticamente significativas entre género y nivel educativo, o género y estudios en la actualidad, mientras que sí las hay entre género y formación profesional, y entre género y capital social (más alto en las mujeres que en los hombres). DISCUSIÓN. A la vista de los datos, sugerimos explorar en mayor medida las dimensiones cualitativas del CS, por lo que recomendamos una metodología de investigación-acción, a fin de incentivar cauces de participación y cooperación entre agencias formativas y comunidades de pescadores y sus familias. Para que el CS tenga efectos tangibles sobre el desarrollo sostenible en estos contextos de vida se necesitan planes coordinados familia-escuela-comunidad. También destacamos algunas acciones estratégicas en términos de educación para el desarrollo.

Palabras clave: Mujer, Capital social, Comunidad, Educación para el Desarrollo, Cooperación. 


\section{Introducción}

Tradicionalmente, la investigación sobre capital social no se ha ocupado mucho de las relaciones de género, incluyendo su manifestación en contextos de pobreza. Cuando lo ha hecho, sin embargo, la preeminencia ha sido para las redes masculinas, sin mostrar las importantes diferencias que es posible encontrar al compararlas con las femeninas. Lo que en estas páginas pretendemos es contribuir a que tal desequilibrio se reduzca y, para ello, nos valemos de un estudio sobre el terreno en el archipiélago caboverdiano. La investigación se realizó entre los años 2011 y 2013.

En el año 2010 la población femenina de Cabo Verde era superior a la masculina en cerca del $1 \%$, pues de los/as 491.683 habitantes del país, 248.260 eran mujeres. La isla de Santiago, donde se sitúa la capital (Cidade da Praia), es la que alberga la mayoría de la población, con 273.919 habitantes (52\% mujeres y 48\% hombres). En la isla hay 27 comunidades pesqueras, lo que representa cerca del $50 \%$ del total nacional (INE, 2010).

Aunque la legislación favorece la equidad de género $^{1}$, incluyendo la existencia de disposiciones de discriminación positiva, los indicadores de participación femenina no son aún los deseables, sobre todo en las comunidades pesqueras. Ellas centran su participación en el plano doméstico, lo que condiciona su implicación en el espacio público. El control que sobre sus relaciones sociales ejercen los maridos se nota, sobre todo, en las comunidades pesqueras de la isla de Santiago.

Sin ir más lejos, las mujeres están limitadas en su nivel de acceso a los recursos financieros y en la gestión que de los mismos pueden hacer en el hogar. Lo cual afecta a la toma de decisiones en materia de salud, educación o relaciones sociales. Todo ello condiciona, desde luego, su participación en los asuntos públicos (Baptista, Brito y Lopes, 2011).
Entre las comunidades de pescadores más pobres del país se encuentran las de la isla de Santiago. Tal situación de pobreza endémica ha tenido su negativo reflejo en términos de presión sobre los recursos naturales, la falta de saneamiento del medio y un débil ejercicio de la ciudadanía a la hora de intentar buscar vías de salida a la problemática del desarrollo en esas comunidades.

A propósito de contextos como el caboverdiano, el "capital social" puede constituirse en un instrumento conceptual y práctico para la promoción de la igualdad, la consolidación de políticas comunitarias adecuadas y para la revitalización de la sociedad civil y de la democracia. En este sentido, el análisis de la relación entre género y capital social de las comunidades de pescadores constituye un tema actual y pertinente, ya que no abundan estudios consistentes en torno a esa relación (Arriagada, 2003; Noguera, 2008). Por ello, en este artículo, buscamos cuantificar el nivel del capital social de las mujeres en las comunidades pesqueras de la isla de Santiago en Cabo Verde, poniendo de relieve la necesidad de una mayor atención a esta relación en pos del desarrollo individual y colectivo de sus miembros.

\section{Promoción de la igualdad y del capital social}

Al concepto de "capital social" le fueron atribuidas múltiples funciones y todas ellas marcan su importancia como elemento clave para explicar y promover el desarrollo económico, mejorar el funcionamiento del sector público, facilitar la gestión en las organizaciones, mantener la estabilidad de los sistemas democráticos y superar la pobreza (Bourdieu, 1980; Coleman, 1987; Portes, 1999).

Como tal, el concepto de "capital social" posee capacidad heurística. Tan importante cualidad epistémica procede, de acuerdo con Portes (1999), de dos fuentes. En primer lugar, se fija 
en las consecuencias positivas de la sociabilidad, situando esas consecuencias en el marco de una discusión más amplia acerca del capital, de modo que advierte acerca de las maneras en que esas formas de relación (no monetarias) generan influencia y poder a la altura de la que puede proporcionar el dinero.

Si consideramos el valor del capital económico, pocas dudas puede haber de que estamos hablando de dinero o de propiedad convertible en moneda. Ahora bien, a la hora de identificar el valor del capital social, lo que conviene es indagar en las normas y valores compartidos por los miembros de la organización social, las redes existentes entre ellos, la cooperación en tareas diversas y, por supuesto, la confianza y reciprocidad que los conecta entre sí. Estos elementos constitutivos del valor del capital social son característicos de la organización social y facilitan la acción conjunta (Correia, 2007). Es posible afirmar que el capital económico produce riqueza, mientras que el rendimiento del capital social se manifiesta en la eficiencia de la sociedad, o de las organizaciones, al facilitar la cooperación entre las personas.

Puestos a definirlo, el capital social sería el conjunto de elementos constituyentes de una sociedad, comunidad u organización que aumentan su eficacia al facilitar la acción conjunta de los individuos en la realización de los objetivos comunes. Estos elementos asumen la forma de normas, redes sociales, confianza generalizada, reciprocidad y valores como la tolerancia y la solidaridad (Correia, 2007). En cualquier caso, redes sociales y normas de reciprocidad no se pueden tener como elementos fijos del capital social pues varían en el tiempo y en el espacio.

Así pues, lo que, en sentido amplio, pone de manifiesto es la contribución de la relacionabilidad entre personas al funcionamiento de la sociedad. Puede decirse que la relacionabilidad interpersonal restrictiva acomoda círculos de influencias, mientras que la relacionabilidad amplia subyacente a la noción de "capital social" (Putnam, 2000), comprende el beneficio de la sociedad en su conjunto. Sin embargo, esta concepción de capital social no ha logrado unanimidad en la comunidad científica, emergiendo divergencias y confusiones conceptuales que no han dejado de dificultar la operacionalidad del concepto.

El origen de la controversia se encuentra en su triple paternidad conceptual (Bourdieu, 1980; Coleman, 1987; Putnam, 2000). Tan ilustres autores utilizan idéntica terminología en sentido no necesariamente coincidente, si bien aluden a elementos del capital social, como los relativos a la confianza, la reciprocidad o las redes sociales y normas, que ejemplifican acuerdos básicos al respecto (Boix y Posner, 1998; Arriagada, 2003). Pero el interés por el capital social también se ha notado en grandes organizaciones económicas como la misma OCDE, que lo ha definido de modo diáfanamente sucinto, a saber, redes y normas, valores y conocimientos compartidos que facilitan la cooperación entre o dentro de los grupos (Helliwell, 2001).

Puesto que el capital social se expresa mediante la conectividad facilitada por la confianza y la participación, la lógica indica la existencia de vínculos directos con los procesos educativos, tanto en sentido extensivo (dinámicas compartidas de logro de niveles de dominio y capacitación que permiten la expedición de credenciales formales) como intensivo (estrategias de aprendizaje interactivo que facilitan el desarrollo de la reciprocidad, la confianza y solidaridad en y desde la experiencia escolar). Hace justo una década que Putnam (2004) planteaba, precisamente, que el número de años de educación formal puede ser un predictor de capital social, ya que un mayor tiempo de educación dilata las redes sociales de los individuos, las hace más fuertes y favorece que esas personas aumenten su participación en sus marcos de vida social y comunitaria. 
Tampoco hay que olvidar que el bagaje educativo de la ciudadanía dista mucho de poder reducirse o agotarse en términos de escolarización institucionalizada. Los espacios educativos son bastante diversos. Está la familia, la comunidad, la empresa o lugar de trabajo, los grupos de iguales, etc. Y ahí lo normal es que la educación potencie la génesis y desarrollo de normas para avanzar en cohesión y solidaridad, o que solicite la estructuración de actividades que permitan compartir y/o cooperar a los individuos del mismo o de distinto grupo étnicocultural y social.

Además, la promoción de la igualdad de oportunidades es primordial para el refuerzo del capital social en las comunidades. En ese sentido, Putnam (2000) ha sido criticado por haber ignorado el problema de la desigualdad como elemento de erosión del capital social. La igualdad es uno de los presupuestos para el desarrollo de la confianza, cuestión central en el concepto de capital social. Lo que ocurre, en definitiva, es que la desigualdad social y política de las personas bloquea su cooperación, sin la cual es ingenuo pensar en la producción de capital social (Correia, 2007).

El desarrollo de derechos sociales es la principal vía para la disminución de las desigualdades que afectan a los niveles de capital social en las comunidades. Puede que el primer paso a dar sea la institución de una renta mínima a fin de asegurar la satisfacción de las necesidades básicas de los individuos y permitirles un ejercicio de la ciudadanía básica. Naturalmente, plantear derechos sociales fundamentales para la promoción de la igualdad y, por extensión, para el desarrollo de comunidades cívicas, supone hablar en serio de educación, de salud y de seguridad, pues de ahí parten las garantías de mínimos para el ejercicio de la ciudadanía (Correia, 2007).

Y está claro, por supuesto, que actuar para reducir desigualdades o ampliar la equidad social es sinónimo de evaluación sólida de la situación que viven las mujeres en grandes partes del mundo.
Hoy, por fortuna, la coincidencia es enorme en cuanto a las ventajas y beneficios asociados a las políticas centradas en la disminución de la desigualdad de género (salud de los niños y niñas, educación infantil, pautas de consumo...) (Banco Mundial, 2005; Noguera, 2008).

En definitiva, la promoción de la igualdad, más allá de ser un imperativo moral, es una condición esencial para el desarrollo porque ha de servir al refuerzo de la cooperación y de la confianza generalizada y, por lo tanto, del capital social.

\section{Planificación del estudio y metodología}

Incluimos en este apartado los aspectos metodológicos básicos del estudio realizado.

\section{Objetivos}

Pretendemos cuantificar el nivel del capital social de las mujeres en las comunidades pesqueras de la isla de Santiago en Cabo Verde, destacando la necesidad de una mayor atención a esta relación en pos del desarrollo individual y colectivo de sus miembros. Para ello nos planteamos como objetivos específicos los siguientes:

- Analizar el nivel educativo de las mujeres en las comunidades pesqueras de la isla de Santiago.

- Estudiar las diferencias en la formación profesional entre hombres y mujeres de las comunidades pesqueras de la isla de Santiago.

- Examinar el índice de capital social de las mujeres de las comunidades pesqueras de la isla de Santiago.

- Valorar el papel que pueden desempeñar las mujeres en el desarrollo sostenible de las comunidades pesqueras de la isla de Santiago. 
Cuadro 1. Variables del estudio

Personales

- Sexo

- Edad

- Tiempo de residencia en

la comunidad estudiada
Escolares

- Nivel educativo

- Formación

- Estudiar/formarse en la actualidad
Familiares

- Medio familiar

- Rendimiento familiar
Capital social

- Sociabilidad e inclusión social

- Confianza y receptividad

- Solidaridad y ayuda

- Participación en acciones voluntarias y cooperación comunitaria

\section{Variables}

Se consideraron distintas variables referidas al ámbito individual, escolar y familiar, además del propiamente vinculado al capital social.

Para medir el capital social se tuvieron en cuenta aquellas relacionadas con las percepciones subjetivas de confianza, la presencia de redes de conexiones, la solidaridad, la inclusión social y la acción voluntaria (Gomes, Gomes y Bueno, 2005; Baptista, Brito y Lopes, 2011).

La elección de tales variables se justifica por el hecho de estar el capital social relacionado con aspectos de la organización social (grupos, redes, normas y confianza), que facilitan formas de cooperación orientadas al beneficio mutuo, con posibilidades de ayudar al desarrollo sostenible de las comunidades pesqueras de la isla de Santiago.

\section{Muestra}

Según el censo de 2010, la estimación de los residentes en las comunidades pesqueras de la isla de Santiago era de 30.032 personas, con edades comprendidas entre los 15 y los 64 años. Se trata de la franja de edad de la población considerada activa por el Instituto Nacional de Estadística de Cabo Verde. Teniendo en cuenta que la tasa de crecimiento anual de la población es del 1,2\%, en tal franja de edad habría en junio de 2011, cuando iniciamos el trabajo de campo, unas 30.580 personas (INE, 2010).

Así, los miembros de las comunidades pesqueras que, finalmente, formaron parte, de la muestra fueron 340 personas, seleccionadas al azar entre la población de esas comunidades.

La distribución por género de los/as encuestados/ as se hizo de forma similar a la registrada para la población total de Cabo Verde y de la isla de Santiago, teniendo en cuenta lo ya referido, esto es, la preeminencia de las mujeres en la población caboverdiana. Es por ello que, de los/as 340 participantes, la mayoría $(51,2 \%)$ son mujeres.

En lo que respecta a la edad, se verifica que más de mitad (54,7\%) están en la edad adulta joven (20-40 años), 24,4\% en la adolescencia (13-20 años), 18,8\% en la edad adulta (40-60 años) y solo el 2,1\%, tiene más de 60 años.

\section{Instrumento}

Como ya hemos señalado, hemos realizado un estudio de encuesta utilizando como instrumento básico un cuestionario adaptado del Banco Mundial (Grootaert, Narayan, NyhanJones y Woolcock, 2002). En este caso, tuvimos que ajustar las preguntas que recogen datos biográficos para ser congruentes con la realidad objeto de estudio. 


\section{Diseño y validación del instrumento}

El cuestionario se estructura en 32 cuestiones, 21 de ellas referidas al capital social y las 11 restantes a informaciones personales. Los aspectos relacionados con el capital social incluyen ítems sobre confianza y receptividad (3), sociabilidad e inclusión social (10), solidaridad y ayuda (3), y participación en acciones voluntarias o cooperación comunitaria (5), y se plantean de forma escalar con cuatro opciones de respuesta. En la segunda parte del cuestionario se recogen datos biográficos, a base de preguntas abiertas y cerradas, tales como sexo, edad, tiempo de residencia en la comunidad, nivel educativo, formación, familia e ingresos.

Una vez elaborado el cuestionario, y para verificar su aplicabilidad, se hizo una prueba piloto con 12 sujetos en la comunidad de Cutelinho (Santa Cruz). En consecuencia, se verificó que: a) todos/as los/as encuestados/as comprendían las cuestiones de la misma forma; b) la lista de opciones de respuesta en la cuestiones cerradas consideraba todas las alternativas para este estudio; c) hubo un elevado grado de aceptación a las cuestiones planteadas, sin que haya ninguna hacia la que los sujetos mostrasen rechazo; d) las cuestiones están ordenadas de forma que no se dan saltos importantes de un asunto a otro; y e) se cuidó que el lenguaje utilizado fuera comprensible para los/as encuestados/as, sin que se evidenciaran problemas o impedimentos al respecto.

Al aplicarse un cuestionario se deben respetar escrupulosamente los procedimientos metodológicos que tienen que ver con la selección de los/as participantes y la administración sobre el terreno.

Es por ello que se explicó a cada uno de los sujetos cuestiones tales como el contexto de la realización del trabajo y los objetivos de la investigación, y se les solicitó la colaboración voluntaria y anónima. Obviamente, se trataba de garantizar la confidencialidad de los datos correspondientes a las personas implicadas en el estudio. Los cuestionarios fueron aplicados a modo de entrevista estructurada, entre la población diana, en siete de los nueve municipios de la isla de Santiago (Ribeira Grande de Santiago, Praia, Santo Domingo, Santa Catarina de Santiago, Santa Cruz, San Miguel y Tarrafal de Santiago) y recogidos por los/as firmantes del trabajo. Cada encuestado/a perteneciente a la muestra fue abordado/a individualmente. Optamos por esta fórmula considerando el bajo nivel educativo en estas comunidades. En Cabo Verde, incluso quienes tienen una habilitación relativamente elevada necesitan de aclaraciones en torno al tema, poco conocido en el país hasta el momento.

Codificación y tratamiento de los datos

Para el tratamiento de los datos se recurrió a la fórmula del Índice del Capital Social (ICS) utilizada por el Banco Mundial en los estudios sobre capital social y desarrollo (Grootaert et al., 2002) y al paquete estadístico SPSS statistics 20.

\section{IMAGEN 1. Fórmula para calcular el Índice del Capital Social (ICS)}

$$
I C S=\frac{1}{n} \sum_{j=1}^{n}\left[\frac{\sum_{i=1}^{m} E_{i j}}{\sum_{i=1}^{m} E_{\max i}}\right]
$$

La contribución de cada indicador (Ci) en el ICS fue calculada con base en la siguiente fórmula:

\section{IMAGEN 2. Fórmula para calcular la contribución} de un indicador en el ICS

$$
C i=\frac{\sum_{i=1}^{n} E i j}{n\left(\sum_{i=1}^{m} E m a ́ x i\right)}
$$

Donde Ci es la contribución del indicador i en la formación ICS. El indicador es un parámetro (o variable) insertado en el cuestionario, o sea, 
una pregunta donde el encuestado podrá responder "siempre (3), a veces (2), nunca (1) o no respondió (0)". Después de proceder con la selección y la cuantificación de las variables representativas de cada indicador, se calculó el índice que mide el capital social de las comunidades pescadoras de la isla de Santiago, así como la participación relativa de cada variable en su composición final.

Ya que el capital social es un componente superestructural, que asociado a los otros capitales puede contribuir al desarrollo sostenible de las comunidades de pescadores de la isla de Santiago, se aplicó la ecuación sobre el desarrollo, muy utilizada por el Banco Mundial, y aplicada por Grootaert et al. (2002), vinculando capital natural $(\mathrm{Kn})$, capital económico (Ke), capital humano (Kh) y capital social (Ks).

El nivel de capital social de las comunidades de pescadores de la isla de Santiago fue medido a través de un índice con el uso de los indicadores que calculan, expresamente, el capital social estructural, cognitivo, de la acción voluntaria, de la solidaridad, de la confianza y de la sociabilidad.

En lo que se refiere a las informaciones personales de los sujetos, las variables edad y rendimiento familiar fueron transformadas bajo forma de intervalos, y las otras en serie numérica.

\section{Análisis e interpretación de resultados}

Con los datos extraídos del cuestionario aplicado, al hacer el cruce de la variable cualitativa género con el nivel de estudios, se constata que solo 2 (1 hombre y 1 mujer) de los 340 encuestados no saben leer ni escribir, y 111 tienen la enseñanza básica incompleta $(54,1 \%$ hombres y $45,9 \%$ mujeres). En lo que se refiere a los 41 que han completado la enseñanza básica, mayoritariamente $(63,4 \%)$ son mujeres. De otro lado, entre los que afirman tener la enseñanza secundaria incompleta destacan los hombres $(52,1 \%)$, porcentaje que se reduce $(46,2 \%)$ en el caso de la enseñanza secundaria completa, donde las mujeres parecen aventajarlos (53,8\%). De los 9 sujetos de la muestra con enseñanza superior, 7 son del sexo femenino y 2 del masculino.

En todo caso, al probar la relación entre ambas variables empleando chi-cuadrado de Pearson se obtiene un valor 6,602 (5 g.l.) y p=0,252, lo que significa que no existen diferencias estadísticamente significativas entre el género y el nivel educativo de los/as encuestados/as; y por ello no se rechaza la hipótesis nula, que afirma que esas variables son independientes $\left[\mathrm{X}_{(262: 5)}^{2}=6,602\right.$; $\mathrm{p}=0,252>0,05]$.

Teniendo en cuenta los datos que acabamos de mencionar, en las comunidades pesqueras de la isla de Santiago, las mujeres poseen un mayor nivel de estudios que los hombres, aunque las diferencias no sean estadísticamente significativas. Resulta extraño, por ello, que no tengan una participación más activa y autónoma en la toma de decisiones, así como en las cuestiones relativas al desarrollo local y comunitario.

Las personas de las comunidades pesqueras de la isla de Santiago tienen auténticas dificultades no solo para acceder sino, fundamentalmente, para concluir sus estudios secundarios y técnico-profesionales, además, claro está, de los de orden superior. La explicación es fácil pues tiene relación con acuciantes problemas socioeconómicos, en especial, el desempleo y el bajo poder adquisitivo. Así pues, es mucho lo que resta por hacer en esas comunidades para que la educación pueda contribuir, como sería esperable, a un sólido equipaje cívico de las personas, de modo que estas se sientan capaces de servir a la sociedad (Correia, 2007).

Al analizar el nivel educativo ${ }^{2}$ de la población en Cabo Verde de 3 o más años en 2010, se concluye que el $43 \%$ tenían solo la enseñanza básica, siendo este porcentaje más reducido en el medio urbano $(39,9 \%)$ que en el medio rural 
$(47,8 \%)$; por su parte, el $31,2 \%$ poseían estudios secundarios y el $12,5 \%$ no tenían instrucción, o sea, nunca frecuentan la escuela $(16,8 \%$ en el rural y $9,9 \%$ en el urbano). El bachillerato o un nivel superior lo habían concluido únicamente el 5,1\%. Tal radiografía se parece a la que arrojan los datos para la isla de Santiago (INE, 2010).

Según los últimos datos disponibles, correspondientes a 2010 (INE 2012), la tasa líquida de escolarización por nivel de enseñanza y medio de residencia era para el medio urbano del 90\% en la enseñanza básica (mujeres 90,4\%; hombres $89,7 \%$ ), $64 \%$ en la secundaria (mujeres $68,3 \%$; hombres $59,7 \%$ ) y $4,8 \%$ en la superior (mujeres 5,5\%; hombres $4,1 \%$ ), mientras que en el rural la situación era del 90,5\% para primaria (mujeres 90,6\%; hombres 90,4\%), $53,4 \%$ para secundaria (mujeres $58,5 \%$; hombres $48,5 \%)$, y $0,8 \%$ en la enseñanza superior (mujeres 0,9\%; hombres 0,8\%).

Como se puede observar en los datos, la situación educativa en el medio rural, al que pertenecen las comunidades pesqueras, es peor que en el medio urbano, aunque son las mujeres las que despuntan en los niveles de educación secundaria.

Al cruzar la variable cualitativa género con la variable cualitativa acerca de si estudia o no en la actualidad, solo 67 de los/as encuestados/as respondieron afirmativamente, sobre todo, hombres. Los/as restantes 273 respondieron que no estaban estudiando, entre los cuales hay mayoritariamente $(53,5 \%)$ mujeres. Se pone de manifiesto, entonces, que hay más hombres estudiando que mujeres. El número tan reducido de personas que estudian puede tener que ver con la edad de los/as integrantes de la muestra.

Además, los resultados de la prueba de independencia entre las dos variables $\left[\mathrm{X}_{(201: 1)}=2,942 ; \mathrm{p}=\right.$ $0,086>0,05$ ] no permiten rechazar la hipótesis nula, ya que esas variables son independientes, lo que nos lleva a afirmar que las diferencias entre los hombres y mujeres que estudian no son significativas.

Justamente, ese mismo número de sujetos $(n=67)$ tienen formación profesional, aunque este grupo está conformado mayoritariamente por mujeres (70,8\%). Esos datos muestran que, en las comunidades pesqueras de la isla de Santiago, hay más mujeres con formaciones profesionales que hombres, sobre todo en las áreas de manipulación y conservación del pescado, y de gestión básica. Esto podría explicarse, en parte, por la poca oferta de acciones de formación destinadas a los profesionales del sector de la pesca. Como ejemplo, en los años 2007 y 2008, para las áreas de manipulación y conservación del pescado, se ofertaron acciones previstas para 160 formandos y solo se contemplaron 59, y en reparación y montaje de redes de pesca solo se cubrieron 26 de las 160 plazas previstas (Silva, 2011).

En todo caso, los resultados de la prueba chicuadrado entre las variables género y formación profesional $\left[\mathrm{X}_{(338: 1)}^{2}=12,355 ; \mathrm{p}=0,000<0,05\right]$ indican que debemos rechazar la hipótesis nula de independencia en ambos casos, lo que significa que existe una relación de dependencia entre género y las variables formación profesional. Es decir, que la diferencia entre hombres y mujeres en la formación profesional es estadísticamente significativa.

Al considerar el género con la realización de alguna acción de formación en la actualidad, tenemos que 27 de los/as encuestados/as sí las realizan, entre los que están, sobre todo, mujeres $(74,1 \%)$. Se constata, por lo tanto, el predominio de los/as que no acuden a acciones formativas (50,5\% hombres y 49,5\% mujeres). Parece, pues, que en esas comunidades, las mujeres muestran más voluntad participativa en las acciones de formación que los hombres.

De acuerdo con Lux-Development (2013: 17), las prioridades de formación tienen que ver, 
sobre todo, con los puestos de conductora, marinero pescador, maestro de pesca, maestro costero y patrón. Se necesita más formación en manejo de equipamiento de navegación, reparación de motores fuera borda, manipulación y conservación del pescado y gestión de pequeñas embarcaciones, formación personal y social, destrezas de salvamento, mantenimiento y reparación de pequeños equipamientos de frío, primeros auxilios y gestión de pequeños negocios. También se precisan más acciones de formación técnico-profesional y superior en diversas áreas.

Tales diferencias entre hombres y mujeres son estadísticamente significativas ya que los datos de chi-cuadrado $\left[\mathrm{X}_{(336: 1)}=5,996 ; \mathrm{p}=0,014<0,05\right]$ nos indican que debemos rechazar la hipótesis nula de independencia entre ambas variables.

Conviene destacar en este punto que los actores del sector pesquero no disponen de suficientes competencias educativas como para asimilar y procesar, convenientemente, la formación técnica. Asimismo, se constata una clara falta de cualificación de los formadores, y en sus acciones lo que acostumbra a aplicarse es una metodología más directiva que participativa, sin que resulte, por supuesto, un clima de familiarización y de comunicación. Todo ello condiciona la discusión y el análisis, bien como ejercicio de las capacidades argumentativas y/o discursivas o, en última instancia, de la asimilación de los contenidos que importan.

De acuerdo con lo que hemos expuesto, el capital social en las comunidades pesqueras de la isla de Santiago alcanza una puntuación de 0,76 , inferior al valor que arroja en las mujeres $(0,77)$ y que es ligeramente superior al de los hombres $(0,74)$. Conviene decir aquí que la desviación típica del capital social en esas comunidades es de 0,101 , con igual valor para las mujeres y de 0,099 para los hombres. El coeficiente de variación es de 13,3\%, ligeramente superior en los hombres $(13,4 \%)$ que en las mujeres $(13,1 \%)$.
Nuestra interpretación de este hecho es que en las comunidades de referencia, el capital social cuenta, en cierta medida, con una base cultural asociada a los roles y a la funcionalidad conductual que exhiben muchas mujeres (cabezas de familia) en sus modos o estilos de prever, gestionar o resolver problemas de la vida cotidiana vinculados a sus labores domésticas o a la frecuente colaboración en el seno de la vecindad. Hay que destacar el aumento experimentado en el número de mujeres "jefes de familia" entre 2000 (40\%), 2007 (45\%) y 2010 (48\%) (INE, 2012).

En el caso femenino, dicho capital alcanza su cota máxima $(0,84)$ en el municipio de Santa Catarina y el mínimo $(0,69)$, en el de Tarrafal. Por el contrario, los hombres registran el máximo $(0,79)$ en las comunidades pesqueras de los municipios de San Domingos, San Miguel y Santa Catarina, y los mínimos en Praia y Tarrafal $(0,68)$. Puede decirse que tales valores son relativamente elevados.

Desde los resultados que arroja la prueba $U$ de Mann-Whitney (MW) al comparar la media del capital social de los/as encuestados/as según el sexo [M-W=11566,000; $p=0,001<0,05]$, es evidente que no hay independencia entre género y capital social, siendo las diferencias en capital social estadísticamente significativas. Concretamente, el nivel de capital social se ha demostrado más alto en las mujeres que en los hombres.

En Cabo Verde, el continuado auge de la emigración, en especial de los hombres que se han ido del país, explica en apreciable medida el gran número de familias encabezadas por mujeres. Sin embargo, en los últimos años, y según el INE (2014), del total de 11.931 emigrantes, entre 2012 y 2013, el 49,3\% eran hombres y $50,7 \%$ mujeres. Mayoritariamente emigraron a Portugal (56,4\%), seguidos de los que se fueron a Estados Unidos (15,4\%), Francia (7,2\%), Brasil (4\%) y China (3\%). Entre las razones para emigrar están la continuación de los estudios (36\%), el reagrupamiento familiar $(21,4 \%)$, la 
búsqueda de trabajo (20\%) y motivos de salud (8\%).

Además, particularmente en las comunidades pesqueras de la isla de Santiago, la práctica de la poligamia ha contribuido a consolidar el fenómeno. Así, para Brito (2011), la pobreza y la falta de hombres en determinadas localidades, junto a la permisividad sexual, la maternidad precoz y una mentalidad abiertamente "machista", han llevado a una proliferación de niños/as "sin padre", a los padres que siempre están "fuera de casa" o al alcoholismo crónico en no pocos casos.

Es bastante frecuente que un hombre mantenga relaciones con dos, o incluso tres, mujeres. Puesto que buena parte de estas mujeres acaban por tener hijos/as, ellas mismas han de asumir un doble rol en relación con su descendencia (Barbe, 2002). Y es lo que ocurre no pocas veces en las comunidades pesqueras de la isla de Santiago. En tal contexto de vida, tampoco son inusuales las mujeres con relaciones, y procreaciones, fuera del matrimonio, lo cual explica, en parte, la asunción, en exclusiva, de las responsabilidades familiares.

Cuando reparamos en el capital social de los/ as encuestados/as, atendiendo al género y fijando nuestra atención en las demarcaciones municipales, lo que se evidencia es la necesidad de implementar políticas públicas adecuadas por parte de las autoridades nacionales y locales, implicando a escuelas, familias y sociedad civil en un soporte conjunto y mancomunado al capital social en todas sus dimensiones, realzando la confianza, la solidaridad, la cooperación, la reciprocidad, la participación en acciones voluntarias o el impulso a los proyectos de desarrollo local y comunitario. Sin olvidar, como tiene apuntado Ander-Egg (2000: 98), la importancia de los nuevos actores sociales - ONG y movimientos sociales-, en esta nueva perspectiva de la acción comunitaria, junto a la relevancia de la misma acción municipal.
Tanto en el país como un todo, como, particularmente, en las comunidades pesqueras de la isla de Santiago, son cada vez más las mujeres que terminan sus estudios y que se implican en acciones de formación. Sin embargo, la educación no parece haber contribuido - como se esperaba hace dos décadas- a mejorar la democracia inclusiva y a la defensa y/o salvaguarda de los derechos humanos, sin cuya visibilidad no es posible afirmar una vida en libertad. Ni siquiera las mujeres, pese a disponer de un nivel educativo y de tasas de capital social relativamente más elevados que los hombres, han conseguido unos niveles de autonomía y de activa participación en el desarrollo de sus comunidades a la altura de las expectativas y circunstancias, teniendo en consideración, sobre todo, los primeros cinco Objetivos de Desarrollo del Milenio contemplados por los países firmantes hasta el 2015: 1) reducir a la mitad la pobreza y el hambre; 2) alcanzar la enseñanza primaria universal; 3) promover la igualdad de género y capacitar a las mujeres; 4) reducir en dos tercios la mortalidad infantil; 5) reducir un $75 \%$ la mortalidad materna; 6) combatir el VIH/SIDA, la malaria y otras enfermedades graves; 7) garantizar la sostenibilidad ambiental; y 8) fortalecer una acción global para el desarrollo (ANMCV, 2010).

No puede extrañarnos, por lo tanto, que en plena vorágine globalizadora uno de los desafíos mayúsculos ante nuestros ojos siga siendo el de apurar las vías y condiciones para una ciudadanía, con paridad e igualdad real entre hombres y mujeres, y esto vale, sin mácula de duda, cuando hablamos de las comunidades pesqueras existentes en la isla de Santiago. En esa realidad, eminentemente rural, la situación de precariedad en las mujeres es un hecho, por más que la de los hombres tampoco se pueda tachar, socioeconómicamente hablando, de envidiable (Melo, 2011).

No obstante, la educación y su permanente mejora sigue erigiéndose como el gran catalizador no ya solo a la hora de optimizar los 
diagnósticos como de afinar, con los medios de que se dispone, el tratamiento de un problema, que va más allá de las relaciones de género, pero cuya gestión efectiva es imposible dejando de lado la cuestión central de cómo las mujeres son pieza indispensable en el desarrollo cívico y democrático de una comunidad y de un país. Es atendiendo a esa "ética del cuidado" cómo creemos que se ha de producir el definitivo ascenso de Cabo Verde en los rankings de desarrollo humano y social, en África y en el mundo.

\section{Discusión y conclusiones}

En este trabajo nos propusimos examinar los niveles de capital social existente en nichos ecológicos tan significativos en el archipiélago atlántico como son los que se vertebran, comunitariamente hablando, en torno a las artes de pesca.

Nos animaba, sobre todo, conocer las diferencias entre hombres y mujeres al respecto para, en su caso, además de usar encuestas pertinentes sobre valores, confianza o reciprocidad entre los/as ciudadanos/as, poder sugerir medidas de corte socioeducativo y alcance político, susceptibles naturalmente de promover ideas y programas de investigación-acción, en el sentido de incentivar cauces de participación y cooperación entre agencias formativas y las comunidades de pescadores y sus familias. Son las dimensiones cualitativas del capital social las que hacen muy recomendable una metodología (participativa) de este tipo, abundando en razones cuando se trata de asuntos educativos o vinculados a la salud en poblaciones pobres (Arriagada, 2003). Porque es muy oportuno indagar las maneras en que su experiencia cotidiana les ha proporcionado cauces de aprendizaje y bases de conocimiento útiles en sus vidas (Bould, Cohen y Walker, 2011).

Hemos podido comprobar las sinergias existentes entre una perspectiva de género y el capital social. Al respecto, también hemos constatado la esencial funcionalidad del capital social como baluarte conceptual en la articulación de un discurso comprensivo acerca de lo que sucede en este tipo de contextos, y aún el análisis de las prácticas relacionales en la vida comunitaria, máxime teniendo en cuenta la economía política que rige, históricamente hablando, las relaciones de género en sociedades sometidas a condiciones de colonización, pero sin pasar por alto el interesante papel de las mujeres en la asunción de responsabilidades y toma de decisiones, pese al control y restricciones impuestas.

De todos modos, la realidad muestra que sin planes coordinados familia-escuela-comunidad y la implicación consciente y directa de los protagonistas en acciones de naturaleza cívica (ethos cultural específico), es poco probable que el capital social tenga efectos tangibles sobre el desarrollo sostenible de estas comunidades.

Pero, teniendo en cuenta lo que nos ha sido posible averiguar sobre género y capital social en las comunidades pesqueras de Cabo Verde, dos son los aspectos que merecen ser destacados por sus posibilidades generadoras de efectos positivos y, tal vez, de acciones estratégicas, en términos de lo que cabe entender por educación para el desarrollo en el referido marco de vida.

El primero de ellos apunta a la necesidad de diseñar políticas públicas que sitúen su epicentro en dinámicas de contrastado empoderamiento de las mujeres caboverdianas, con el propósito de incrementar su asertividad y el uso de sus capacidades y destrezas en el manejo de situaciones familiares y comunitarias, considerando patrones normativos en individuos y grupos.

Y el segundo, como derivación práctica del anterior, se debería orientar hacia la sistematización de las dimensiones más relevantemente vinculadas al capital social de las mujeres en esas comunidades pesqueras ya que, teniendo 
presentes los datos que aporta el trabajo de indagación realizado, podrían ser la base de un plan educativo que enfatizaría la formación social y profesional de hombres y mujeres a la luz de necesidades asociadas a carencias y déficits que ponen en peligro la supervivencia económica, la optimización de los lazos interpersonales o ambas cosas a la vez.

\section{Notas}

${ }^{1}$ A título de ejemplo, la Ley 84/VII/2011, de 10 de enero, establece las medidas destinadas a prevenir y reprimir el crimen de violencia basada en el género.

${ }^{2}$ De acuerdo con el art. 12 del Decreto-Legislativo 2/2010, de 7 de mayo, que define las Bases del Sistema Educativo, este comprende los subsistemas de la educación preescolar (4-6 años), de la educación escolar y de la educación extraescolar. La educación escolar incluye los subsistemas de la enseñanza básica, siendo este nivel de enseñanza universal, obligatorio y gratuito, con duración de 8 años, secundario ( 4 años) y superior, así como modalidades especiales de enseñanza. La educación extraescolar engloba las actividades de alfabetización, posalfabetización y formación profesional. El sistema educativo integra también la formación técnico-profesional y se articula estrechamente con el sistema nacional de formación y aprendizaje profesional.

\section{Referencias bibliográficas}

Ander-Egg, E. (2000). Metodología y práctica del desarrollo de la comunidad 2. Buenos Aires: Grupo Editoral Lumen Hvmanitas.

ANMCV (Associação Nacional dos Municípios Cabo-verdianos) (2010). Matriz de Convergência dos Municípios Cabo-verdianos para os ODM. Praia: ANMCV.

Arriagada, I. (2003). Capital social: potencialidades y limitaciones analíticas de un concepto. Estudios Sociológicos, 21 (3), 557-584.

Banco Mundial (2005). Informe sobre el desarrollo mundial 2006: Equidad y Desarrollo. Washington, D. C.: Banco Mundial.

Baptista, A., Brito, O., y Lopes, J. (2011). Capital social e desenvolvimento sustentável das comunidades piscatórias de Cabo Verde. En J. Brito (coord.), Colectânea Ciencia Regional em Cabo Verde: Desenvolvimento e Planeamento (pp. 45-57). Cascais: Principia Editora.

Barbe, A. (2002). Les îles du Cap-Vert: de la découverte à nous jours, une introduction. S/l: LHarmattan.

Boix, C., y Posner, D. (1998). Social capital: explaining its origins and effects on government performance. British Journal of Political Science, 4, 686-693.

Bould, D., Cohen, R., y Walker, D. (eds.) (2011). El aprendizaje a partir de la experiencia. Interpretar lo vital y cotidiano como fuente de conocimiento. Madrid: Narcea.

Bourdieu, P. (1980). Le capital social. Actes de la Recherche en Sciences Sociales, 31, 2-3.

Brito, J. (coord.) (2011). Colectânea Ciencia Regional em Cabo Verde: Desenvolvimento e Planeamento. Cascais: Principia Editora.

Coleman, J. (1987). Norms as social capital. En G. Radnitzky y P. Bernholz (eds.), Economic imperialism: the economic method applied outside the field of economics (pp. 133-153). New York: Paragon House Publishers.

Correia, S. (2007). Capital social e comunidade cívica, o círculo vicioso da cidadania: aplicação do modelo de Putnam aos residentes do bairro de Caselas. Lisboa: Edições Instituto Superior de Ciências Sociais e Políticas - Universidade Técnica de Lisboa. 
Mujer y capital social en las comunidades pesqueras de Cabo Verde

Gomes, A., Gomes, A., y Bueno, N. (2005). Capital social comunitário: um estudo em assentamento rural. En Anais do XLIII Congresso da Sociedade de Economia e Sociologia Rural (SOBER), Ribeirão Preto.

Grootaert, C., Narayan, D., Nyhan-Jones, V., y Woolcock, M. (2002). Cuestionario integrado para la medición del capital social. Washington, D.C.: Banco Mundial.

Helliwell, J. (ed.) (2001). The contribution of human and social capital to sustained economic growth and well-being. Ottawa: HRDC y OECD.

Instituto Nacional de Estatística de Cabo Verde (2010). Recenseamento Geral de População e Habitação de 2010. Praia: INE-CV.

Instituto Nacional de Estatística de Cabo Verde (2012). Mulheres e homens em Cabo Verde: factos e número. Praia: INE-CV.

Instituto Nacional de Estatística de Cabo Verde (2014). Dados sobre a emigração cabo-verdiana. Praia: INE-CV.

Lux-Development (2013). Estudo/relatório do sector da família profissional Marítimo Pesqueiro. Praia: Lux-Development.

Melo, M. (2011). Educação, formação e qualificação profissional da mulher rural cabo-verdiana: pressupostos básicos para o desenvolvimento. En C. Silva y C. Fortes (Orgs.), As mulheres em Cabo Verde: experiências e perspectivas (pp. 127-138). Praia: CIGEF/UniCV.

Noguera, P. (coord.) (2008). Capital social, género y desarrollo. Murcia: Ed. de la Universidad de Murcia.

Portes, A. (1999). Capital social: sus orígenes y aplicaciones en la sociología moderna. En J. Carpio e I. Novakovsky (comps.), De igual a igual. El desafio del Estado ante los nuevos problemas sociales (pp. 243-266). Buenos Aires: Fondo de Cultura Económica.

Putnam, R. (2000). Bowling alone: the collapse and revival of american community. New Yok: Touchstone.

Putnam, R. (2004). Education, diversity, social cohesion and social capital. Meeting of OECD Education Ministers. Raising the quality of learning for all, March, Dublin.

Silva, O. (2011). Formação de operadores e técnicos do sector das pescas. En J. Nascimento, A. Martins y A. Medina (coords.), Colectânea do Seminário sobre o Sector das Pescas (pp. 83-88). Mindelo: SIRPD y DIHA.

Abstract

Women associal capital in the Cape Verde fishing communities

INTRODUCTION. Little or no attention has been paid to research studies on social capital (SC) based on female networks.. To this end, we make use of a field study in Cape Verde focusing on the Santiago Island fishing communities. METHOD. A survey of cross-sectional descriptive quantitative methodology was employed using a questionnaire. The sample consisted of 340 people, selected at random among members of the communitiy that ranged from 15 to 64 years of age. In order to measure the Social Capital Index (SCI), variables related to perceptions of trust, networks, solidarity, social inclusion, and voluntary action were taken into account. RESULTS. There are no statistically significant differences between gender and the level of education, or gender and current studies, but there are differences between gender and Vocational Training, and between gender and social capital. These differences were significantly higher in women than in men. DISCUSSION. In view of these data, we suggest further studies on the qualitative dimensions of SC, applying an action research methodology, with the aim of encouraging participation and cooperation channels among training institutions and the fishing communities and 
their families. Coordinated family-school-community plans are needed so that SC could have a tangible impact on sustainable development in these contexts of life. We also point out some strategic actions in terms of education for development.

Keywords: Woman, Social capital, Community, Education for development, Cooperation.

\section{Résumé}

La femme et le capital social dans les communautés de pêche de Cap-Vert

INTRODUCTION. L'objectif de ce travail est de contribuer à la diminution du déséquilibre dans l'assistance prêtée pour la recherche sur le capital social (CS) aux réseaux associatifs au détriment des féminins. Pour cela, nous avons réalisé une étude sur le terrain à Cap-Vert, visée aux communautés de pêche de l'île de Santiago. METHODE. Nous avons utilisé une méthodologie quantitative du type descriptif à travers de la réalisation d'enquêtes par questionnaire. Leéchantillon a été composé par 340 personnes, lesquelles ont été choisies au hasard parmi les membres des communautés dont leur âge était compris entre 15 et 64 ans. Pour mesurer l'Indice de Capital Social (ICS), on a pris en compte des variables liées aux perceptions de la confiance, des réseaux, de la solidarité, de l'inclusion sociale et de l'action bénévole. RESULTATS. Dans l'actualité es différences importantes d'après un point de vu statistique ne sont pas obtenues ni en relation au genre et le taux d'éducation ni entre le genre et les études, tandis qu'elles apparaissent entre le genre et la formation professionnelle et entre le genre et le capital social (ce qui est plus élevé dans les femmes que dans les hommes). DISCUSSION. Vues les données, nous proposons l'examen à grande échelle des dimensions qualitatives du CS; pour cela, nous recommandons une méthode de recherche-action pour stimuler les voies de participation et de coopération entre les agences formatives et les communautés de pêche et leurs familles. Des plans coordonnés entre les familles, les écoles et les communautés sont nécessaires pour que le CS ait des effets tangibles sur le développement durable dans ces contextes de vie. Finalement, nous soulignons de certaines actions stratégiques dans le domaine de l'enseignement pour le développement.

Mots clés: Femme, Capital social, Communauté, Enseignement pour le développement, Coopération.

\section{Perfil profesional de los autores}

\section{Simao Paulo Rodrigues Varela}

Profesor de la Universidade Jean Piaget de Cabo Verde. Máster en Derecho (Universidade do MinhoPortugal), postgraduación en Ordenamiento del Territorio (Universidade de Cabo Verde/Universidade Federal de Rio Grande do Sul-Brasil). Doctor en Ciencias de la Educación (Universidad de Santiago de Compostela). Coordinador de la licenciatura en Administración Local, coordinador del Máster en Políticas Públicas y Administración Local. Miembro de los Consejos General y Científico de la Universidad J. Piaget de Cabo Verde.

Correo electrónico de contacto: spaulo45@hotmail.com 


\section{Miguel A. Santos Rego (autor de contacto)}

Catedrático de Universidad, Premio Nacional de Investigación Educativa. Ha publicado numerosos trabajos en España y en el extranjero. Sus líneas de investigación se centran en educación y pedagogía intercultural, aprendizaje cooperativo, educación y desarrollo de la sociedad civil y políticas educativas. Es coordinador del Grupo de Investigación Esculca-USC y de la Red de Investigación RIES. Tiene reconocidos cinco (5) Sexenios (CNEAI).

Correo electrónico de contacto: miguelangel.santos@usc.es

Dirección para la correspondencia: Facultad de Ciencias de la Educación. Campus Vida. Universidad de Santiago de Compostela, 15782 Santiago de Compostela.

\section{María del Mar Lorenzo Moledo}

Profesora titular de universidad. Pertenece al Grupo de Investigación Esculca-USC y a la Red de Investigación RIES. Premio Nacional de Investigación Educativa. También ha recibido el Premio María Barbeito de Educación en Galicia a la Investigación Pedagógica en su tercera edición y publicado numerosos artículos en revistas de acreditado prestigio científico tanto nacionales como internacionales, además, es coautora de varios libros. Es la directora de la Cátedra UNESCO-USC de Cultura de Paz y Derechos Humanos.

Correo electrónico de contacto: mdelmar.lorenzo@usc.es 